\title{
Outcome of Endoscopic Ultrasound-Guided Sampling of Mediastinal Lymphadenopathy
}

\author{
Tae Young Park $\mathbb{D}^{1}$ and Jeong Seop Moon $^{2}$ \\ ${ }^{1}$ Division of Gastroenterology, Chung-Ang University College of Medicine, Seoul, Republic of Korea \\ ${ }^{2}$ Department of Internal Medicine, Inje University Seoul Paik Hospital, Seoul, Republic of Korea \\ Correspondence should be addressed to Tae Young Park; ptymd@hotmail.com
}

Received 6 November 2021; Revised 7 February 2022; Accepted 16 February 2022; Published 7 March 2022

Academic Editor: Jose Celso Ardengh

Copyright (C) 2022 Tae Young Park and Jeong Seop Moon. This is an open access article distributed under the Creative Commons Attribution License, which permits unrestricted use, distribution, and reproduction in any medium, provided the original work is properly cited.

\begin{abstract}
Background and Objectives. Endoscopic ultrasound (EUS)- guided transesophageal fine needle biopsy has been used as a method for histologic evaluation of mediastinal lymph nodes (LNs). This study aimed to compare the outcomes of the EUS-guided sampling with mediastinal lymphadenopathy using a 19-gauge trucut needle and 22-gauge fine needle aspiration (FNA) needle. Methods. From May 2006 to January 2017, patients with mediastinal lymphadenopathy, who received an EUS-guided trucut biopsy or an FNA biopsy, were retrospectively reviewed. Demographic data, endosonographic characteristics of LNs including size, shape, border, echotexture, and echogenicity, diagnostic yield, and adverse events between the trucut needle group and aspiration needle group were compared. Results. A total of 69 patients (trucut group, $n=33$ vs. aspiration group, $n=36$ ) were identified. There were no significant differences in demographic data, indication for an EUS-guided biopsy, location of LNs, number of needle passes, and endosonographic features of LNs between the two groups. The sizes of LNs were larger in the trucut group than in the aspiration group $(28.9 \pm 14.0 \mathrm{~mm}$ vs. $21.1 \pm 8.8 \mathrm{~mm}, P=0.007)$. However, there was no significant difference in the ratio of LNs that were $\geq 10 \mathrm{~mm}$ in both groups. The overall accuracy of the EUS-guided biopsy for the diagnosis of malignant lesions was 79.7\% (55/69). There were no significant differences in the histological diagnostic yield of malignant LNs between the two groups. There were no significant procedure-related adverse events in both groups. Conclusion. The EUS-guided biopsy can be a useful method for histologic evaluation of mediastinal nodal lesions.
\end{abstract}

\section{Introduction}

Mediastinal lymph nodal staging is the most important consideration when selecting the optimal treatment strategy for lung cancer. In a lung cancer nodal staging work up, endobronchial ultrasound (EBUS)- guided transbronchial needle aspiration is preferred for evaluating mediastinal lymph nodal stages $[1,2]$ In centers in which EBUS is unavailable, mediastinal lymph node (LN) excision using mediastinoscopy under general anesthesia can be performed. However, excision biopsy using mediastinoscopy can be an expensive and invasive procedure, and it can be associated with a prolonged hospital stay or postoperative complications such as pneumonia and mediastinitis [3]. All centers do not have EBUS equipment, or EBUS is not always available. There- fore, endoscopic ultrasound (EUS)- guided transesophageal fine needle aspiration (FNA) in mediastinal lymphadenopathy is the only alternative method of histologic confirmation without surgical intervention.

In addition, in diseases other than lung cancer that can cause mediastinal lymphadenopathies, including tuberculosis, esophageal cancer, lymphoma, and sarcoidosis, the EUS-guided tissue acquisition of mediastinal LNs is a suboptimal diagnostic method for histologic confirmation that supports a specific treatment including antituberculous therapy and chemotherapy, cytotoxic therapy, or immunosuppressive therapy $[4,5]$.

Currently, in most of large volume centers where EBUS is available, the EBUS-guided transbronchial mediastinal LN biopsy is a standard method for tissue acquisition of 
mediastinal LNs. However, in some centers, where EBUS is not available, the EUS-guided transesophageal mediastinal LN fine needle biopsy or aspiration is still used as a suboptimal diagnostic method [6]. This study was aimed at evaluating clinical outcomes of the EUS-guided biopsies of mediastinal lymphadenopathy by comparing a 19-gauge trucut needle group and 22-gauge FNA needle group.

\section{Methods}

2.1. Patients. From May 2006 to January 2017, patients with mediastinal lymphadenopathy who received an EUS-guided trucut biopsy or a FNA biopsy at Inje University Seoul Paik Hospital were retrospectively reviewed. This study was approved by the Institutional Review Board of the Inje University Seoul Paik Hospital (IRB No. PAIK 2018-10-005-001).

2.2. Endoscopic Procedure. Written informed consent for the EUS-guided tissue acquisition of mediastinal lymphadenopathy was obtained from all of the patients before the procedures. All of the patients underwent EUSguided trucut biopsy or FNA biopsy in one session. The procedures were performed under conscious sedation in a left lateral position using midazolam ( $0.05 \mathrm{mg}-0.1 \mathrm{mg} /$ $\mathrm{kg}$ ) and/or propofol $(0.5 \mathrm{mg}-1 \mathrm{mg} / \mathrm{kg})$. The analgesic agent used was intravenous meperidine $(25 \mathrm{mg})$. To control duodenal peristalsis, intravenous hyoscine-N-butylbromide was routinely administered. Intravenous third-generation cephalosporin was administered prophylactically before the endoscopic procedure. An anterior oblique-viewing linear array echoendoscope (UCT 240; Olympus Optical Co. Ltd., Tokyo, Japan) was used in the procedure. The procedure was performed by one experienced endosonographer (J.H.L.).

Endosonographic characteristics including size $(\geq 10 \mathrm{~mm}$ vs. $<10 \mathrm{~mm}$ ), shape (round vs. elongated, ellipsoidal, or triangle), border (sharp demarcated vs. fuzzy), echotexture (homogeneous vs. heterogeneous), and echogenicity (hypoechoic vs. hyperechoic, isoechoic, or mixed echoic) of LNs were evaluated with the B-mode of EUS [7]. After endosonographic evaluation of the mediastinal LNs, tissue sampling from the lesions was performed under EUS guidance. Either a 19-gauge trucut biopsy needle (Quick-Core ${ }^{\circledR}$, Endoscopic Ultrasound Needle; Cook Endoscopy, Winston-Salem, NC, United States) or a 22-gauge FNA needle (EchoTip ${ }^{\circledR}$ Ultra Endoscopic Ultrasound Needle; Cook Endoscopy) was selected for the procedure at the discretion of the endoscopist (J.H.L.).

2.3. Outcomes. The primary outcome was to evaluate the efficacy according to the needle type by comparison of diagnostic yield including accuracy, sensitivity, specificity, positive predictive value (PPV), and negative predictive value (NPV). The secondary outcome was to compare the safety by comparison of procedure-related adverse events including bleeding, perforation, and mediastinitis by needle type.
TABLE 1: Baseline characteristics of the study cohort $(n=69)$.

\begin{tabular}{lc}
\hline Characteristics & Value \\
\hline Age (y), mean \pm SD & $63.3 \pm 13.6$ \\
Male gender, $n(\%)$ & $51(73.9)$ \\
Indications of procedure, $n(\%)$ & \\
Lung cancer & $52(75.4)$ \\
Tuberculosis & $6(8.7)$ \\
Esophageal cancer & $3(4.3)$ \\
Sarcoidosis & $2(2.9)$ \\
Lymphangioma & $2(2.9)$ \\
Lymphoma & $1(1.4)$ \\
UIP & $1(1.4)$ \\
Atypical pneumonia & $1(1.4)$ \\
Recurred metastatic LAP of stomach cancer & $1(1.4)$ \\
Location of lymph nodes, $n$ (\%) & \\
Subcarinal & $57(82.6)$ \\
Aortopulmonary window & $8(11.6)$ \\
Paratracheal & $3(4.3)$ \\
Paraesophageal & $1(1.4)$ \\
Size of lymph nodes (mm), mean \pm SD & \\
Long axis & $24.8 \pm 12.1$ \\
Short axis & $13.6 \pm 6.5$ \\
\hline SD: standard deviation; UIP: usual interstitial & pneumonitis; LAP: \\
lymphadenopathy. &
\end{tabular}

2.4. Statistical Analysis. The categorical variables were compared using a chi-squared test, and the continuous variables were compared using Student's $t$-test. A comparison of accuracy, sensitivity, specificity, PPV, and NPV by needle type was performed with the $95 \%$ confidence interval. A $P$ value $<0.05$ was considered statistically significant. All statistical analysis was performed with IBM SPSS statistics software, version 25.0 (IBM Corp., Armonk, NY).

\section{Results}

A total of 69 patients were identified. The mean age of the study cohort was $63.3 \pm 13.6$ years, and 51 patients (73.9\%) were of male gender. Indications for the EUSguided biopsies were lung cancer in 52 cases $(75.4 \%)$, tuberculosis in 6 cases $(8.7 \%)$, esophageal cancer in 3 cases (4.3\%), sarcoidosis in 2 cases $(2.9 \%)$, lymphangioma in 2 cases $(2.9 \%)$, lymphoma in 1 case $(1.4 \%)$, usual interstitial pneumonitis in 1 case $(1.4 \%)$, atypical pneumonia in 1 case $(1.4 \%)$, and recurred metastatic lymphadenopathy of stomach cancer in 1 case $(1.4 \%)$. Locations of LNs were in the subcarinal in 57 cases $(82.6 \%)$, in the aortopulmonary window in 8 cases $(11.6 \%)$, in the paratracheal in 3 cases $(4.3 \%)$, and in the paraesophageal in 1 case $(1.4 \%)$. The baseline characteristics of the study cohort are summarized in Table 1.

Among 69 cases of the EUS-guided mediastinal LN biopsy, 33 cases were performed with a trucut needle and 
TABLE 2: Characteristics by needle type.

\begin{tabular}{|c|c|c|c|}
\hline & Trucut $(n=33)$ & Aspiration $(n=36)$ & $P$ value \\
\hline Age, mean \pm SD & $60.7 \pm 14.3$ & $65.6 \pm 12.6$ & 0.132 \\
\hline Gender, $n(\%)$ & & & 0.523 \\
\hline Male & $24(72.7)$ & $27(75.0)$ & \\
\hline Female & $9(27.3)$ & $9(25.0)$ & \\
\hline Indication of biopsy, $n(\%)$ & & & 0.784 \\
\hline Lung caner & $22(66.7)$ & $30(83.3)$ & \\
\hline Tuberculosis & $4(12.1)$ & $2(5.6)$ & \\
\hline Esophageal cancer & $2(6.1)$ & $1(2.8)$ & \\
\hline Lymphoma & $1(3.0)$ & 0 & \\
\hline Sarcoidosis & $1(3.0)$ & $1(2.8)$ & \\
\hline Others* & $3(9.1)$ & $2(5.6)$ & \\
\hline \multicolumn{4}{|c|}{ Size of lymph node $(\mathrm{mm})$, mean \pm SD } \\
\hline Long axis & $28.9 \pm 14.0$ & $21.1 \pm 8.8$ & 0.007 \\
\hline Short axis & $15.9 \pm 1.1$ & $11.4 \pm 5.8$ & 0.003 \\
\hline Location of lymph node, $n(\%)$ & & & 0.829 \\
\hline Subcarinal & $26(78.8)$ & $31(86.1)$ & \\
\hline Aortopulmonary window & $4(12.1)$ & $4(11.1)$ & \\
\hline Paratracheal & $2(6.1)$ & $1(2.8)$ & \\
\hline Paraesophageal & $1(3.0)$ & 0 & \\
\hline Number of needle pass, $n(\%)$ & & & 0.796 \\
\hline 1 & $24(72.7)$ & $27(75.0)$ & \\
\hline 2 & $4(12.1)$ & $3(8.3)$ & \\
\hline 3 & $3(9.1)$ & $4(11.3)$ & \\
\hline 4 & $1(3.0)$ & $2(5.6)$ & \\
\hline 5 & $1(3.0)$ & 0 & \\
\hline
\end{tabular}

*Lymphangioma $(n=2)$, usual interstitial pneumonitis $(n=1)$, pneumonia $(n=1)$, and recurred metastatic lymphadenopathy of stomach cancer $(n=1)$.

36 cases were performed with an aspiration needle (trucut group, $n=33$ vs. aspiration group, $n=36)$. A comparison of characteristics between the two groups is presented in Table 2. There were no significant differences in age, gender, indication of the EUS-guided biopsy, location of LNs, and the number of needle passes between the two groups. The long axes of target LNs were larger in the trucut group than the aspiration group $(28.9 \pm 14.0 \mathrm{~mm}$ vs. $21.1 \pm 8.8 \mathrm{~mm}, P$ $=0.007)$. The short axis of the LNs was also larger in the trucut group than the aspiration group $(15.9 \pm 1.1 \mathrm{~mm}$ vs. $11.4 \pm 5.8 \mathrm{~mm}, P=0.003)$. However, there was no significant difference in the ratio of LNs that were $\geq 10 \mathrm{~mm}$ in both groups. Endosonographic features of LNs, including size, shape, border, echotexture, and echogenicity, of the two groups are shown in Table 3. There were no significant differences between two groups in terms of endosonographic features. Two cases of the EUS-guided biopsy of mediastinal lymph node were shown in Figures 1 and 2.

Diagnostic yield for detecting malignant lesions of the total study cohort and comparison by needle type is summarized in Table 4. Overall accuracy of the EUS-guided biopsy for the diagnosis of malignant nodal lesions was 79.7\% (55/ $69)$. In subgroup comparison, the accuracy, sensitivity, and
NPV were higher in the trucut group than the aspiration group. However, there was no statistical significance between the two groups. Specificity and PPV were $100 \%$ in both groups. There were no significant procedure-related adverse events in both groups.

\section{Discussion}

Tissue acquisition of mediastinal LNs is a critical procedure that is performed to determine the treatment plan in lung cancer as well as tuberculosis, esophageal cancer, lymphoma, and sarcoidosis, which can cause mediastinal lymphadenopathies. There have been many invasive techniques for tissue sampling of mediastinal LNs including computed tomography (CT)- guided percutaneous biopsy, mediastinoscopy, mediastinotomy, thoracoscopy, EBUS-guided biopsy, and EUS-guided biopsy [8]. Currently, the EBUS and EBUSguided biopsies are widely used and considered as essential procedures for the evaluation of mediastinal nodal lesions in lung cancer staging work ups. An EBUS-guided transbronchial approach can be useful for sampling the highest mediastinal (station 1), upper and lower paratracheal (stations 2 and 4), and hilar LNs (station 10), as well as 
subcarinal LNs (station 7) $[3,8]$. However, the EBUS-guided biopsy is not readily available due to regions of LN stations or the absence of a dedicated endoscope for EBUS. Mediastinoscopy is performed under general anesthesia in the operating room. Mediastinoscopy has been demonstrated to be effective and safe in mediastinal nodal staging but can be associated with postoperative morbidity and mortality and high cost, and it may be a cumbersome process. Therefore, the EUS-guided biopsy under conscious sedation can be a minimally invasive, safe, and well-tolerated alternative method to mediastinoscopy. An EUS-guided transesophageal approach can be particularly useful for evaluation of the inferior pulmonary ligament (station 9), esophageal (station 8), subcarinal (station 7), and aortopulmonary window (station 5) LNs, which are difficult to access via mediastinoscopy $[3,8]$. Furthermore, the EUS-guided mediastinal lymph node biopsy can be useful for paraesophageal LNs (station 8) or pulmonary ligament LNs (station 9) because common EBUS cannot reach these lymph nodes due to the location.

The EUS-guided FNA was first introduced in 1992 for the evaluation of submucosal or ulcerative lesions of the upper or lower GI tract that are suspicious for malignancy but negative on conventional forceps biopsy [9]. Currently, the EUS-guided FNA has been widely used in pathologic diagnosis of intra-abdominal and mediastinal diseases ranging from malignant lesions to benign lesions [10-15]. Although diagnostic accuracy of the EUS-guided FNA generally has been accepted to be high, the result can be inconclusive. A large amount of tissue is needed for differential diagnosis such as tissue architecture or immunohistochemistry [6]. Sometimes, it is difficult to interpret the results due to blood contamination, necrotic material, or inflammatory cells. Therefore, it would need on-site cytologic evaluation or an additional session of the EUS-guided FNA, and this could be a laborious process. To overcome these limitations, the EUS-guided trucut biopsy using a larger needle caliber was introduced in 2002 [16]. The diagnostic accuracy of the EUS-guided trucut biopsy has been considered to be compatible with those of the EUS-guided FNA [17, 18]. However, because of difficulty of manipulation due to rigidity of the needle and issues of safety due to a spring-loaded firing mechanism, the use of the EUS-guided trucut biopsy has been decreased and practically limited, not only for mediastinal lesions but also pancreaticobiliary lesions [19, 20]. The reverse bevel needle (ProCore ${ }^{\circledR}$ Endoscopic Ultrasound Needle; Cook Endoscopy) was introduced into endoscopic practice, and trucut needle has been replaced into reverse bevel needle. Recently, newer end-cutting FNB needle such as fork-tip design with 2 leading sharp tips (SharkCore ${ }^{\mathrm{TM}}$, Medtronic, Minneapolis, Minn) and 3 symmetric cutting edges (Acquire ${ }^{\mathrm{TM}}$, Boston Scientific Corp, Natick, Mass) has been also introduced and has been increasingly used $[21,22]$.

In a most recent study comparing the EUS-guided fine needle biopsy (FNB) vs. EUS-guided FNA in abdominal LN lesions, diagnostic accuracy was significantly higher in the EUS-FNB group as compared to the EUS-FNA group
TABLE 3: Endosonographic feature of lymph nodes.

\begin{tabular}{lccc}
\hline & $\begin{array}{c}\text { Trucut } \\
(n=33)\end{array}$ & $\begin{array}{c}\text { Aspiration } \\
(n=36)\end{array}$ & $\begin{array}{c}P \\
\text { value }\end{array}$ \\
\hline $\begin{array}{l}\text { Size, } \mathrm{n}(\%) \\
\geq 10 \mathrm{~mm}\end{array}$ & $33(100)$ & $35(97.2)$ & 0.335 \\
$\quad<10 \mathrm{~mm}$ & 0 & $1(2.8)$ & \\
Shape, $n(\%)$ & & & 0.214 \\
$\quad$ Round & $19(57.6)$ & $16(44.4)$ & \\
$\quad$ Elongated or & $10(30.3)$ & $18(50.0)$ & \\
ellipsoidal & $4(12.1)$ & $2(5.6)$ & \\
$\quad$ Triangle & & & 0.453 \\
Border, $n$ (\%) & $28(84.8)$ & $28(77.8)$ & \\
$\quad$ Sharp demarcated & $5(15.2)$ & $8(22.2)$ & \\
$\quad$ Fuzzy & & & 0.528 \\
Echotexture, $n(\%)$ & $19(57.6)$ & $18(50.0)$ & \\
$\quad$ Homogeneous & $14(42.4)$ & $18(50.0)$ & \\
$\quad$ Heterogeneous & & & 0.110 \\
Echogenicity, $n(\%)$ & $16(48.5)$ & $12(33.3)$ & \\
Hypoechoic & $12(36.4)$ & $10(27.8)$ & \\
Mixed echoic & $3(9.1)$ & $12(33.3)$ & \\
Isoechoic & $2(6.1)$ & $2(5.6)$ & \\
Hyperechoic & & & \\
\hline
\end{tabular}

(87.62\% versus $75.24 \%, P=0.02)$. These findings suggested that EUS-FNB can be preferred to EUS-FNA for sampling intra-abdominal LNs in patients with suspected malignancy [23].

In the current retrospective study conducted at our center, at which EBUS and EBUS-guided mediastinal nodal biopsy are unavailable, the EUS-guided biopsy of mediastinal lymph nodes using a trucut needle or FNA needle is reported. Overall, accuracy of the EUS-guided transesophageal LN biopsy for histologic confirmation was $79.7 \%$ (55/69). There were no significant differences in the diagnostic yield by needle type. No significant procedure-related adverse events occurred in both groups.

There are several limitations of note in this study. First, because the EBUS-guided biopsy is unavailable at our center, comparative study regarding this issue could not be performed. Second, trucut needle is commercially unavailable due to safety problem; the findings of this study cannot generally be applied for evaluation of mediastinal lymphadenopathy in clinical practice. Third, current retrospective study was performed using old database in single low volume center. Over the last 10 years, novel FNB needle designs have been developed. 19G Trucut needle was used for acquisition of histological tissue samples for immunohistochemistry at our center. However, 19G trucut needle is not flexible, and its stiffness might render difficulty of sampling in certain situations. Difficulty of manipulation and safety issue limited the use of trucut needle in clinical practice. Nowadays, it has been replaced to ProCore ${ }^{\circledR}$ needle or 


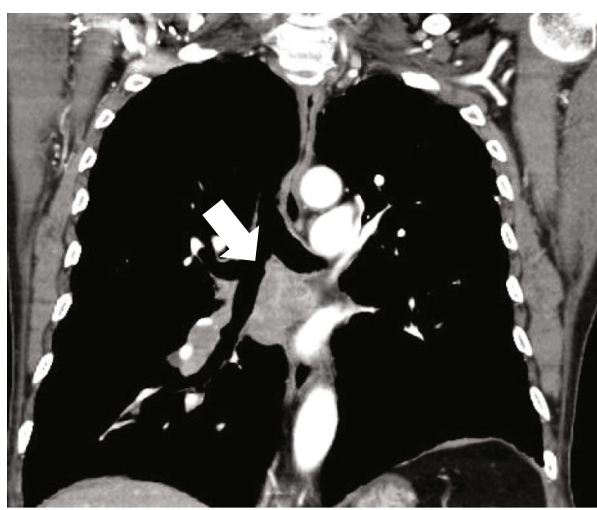

(a)

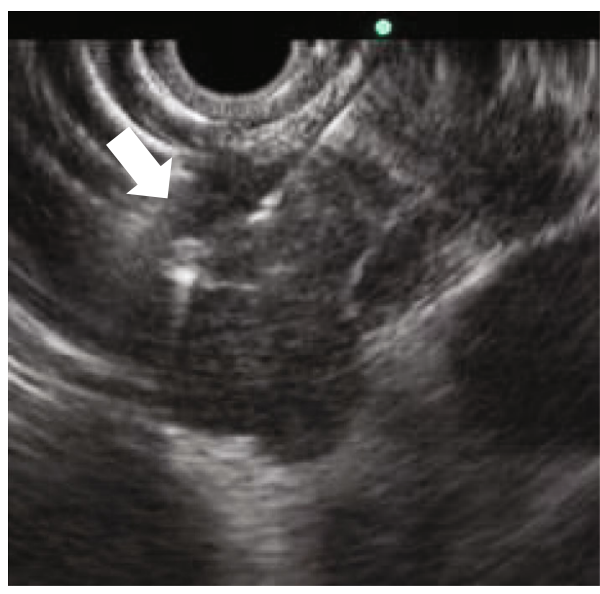

(b)

FIGURE 1: A 78-year-old male patient. (a) Subcarinal lymphadenopathy (arrow) is shown on CT scan. (b) On EUS view, multiple round shaped and sharp-demarcated lymph nodes (arrow) were conglomerated in station 7. The EUS-guided biopsy was performed. CT: computed tomography; EUS: endoscopic ultrasound.

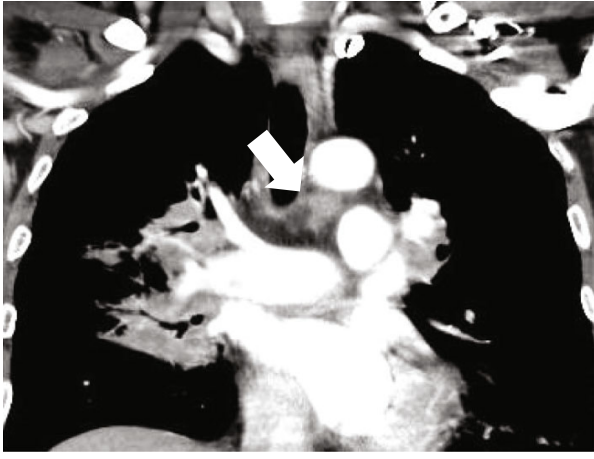

(a)

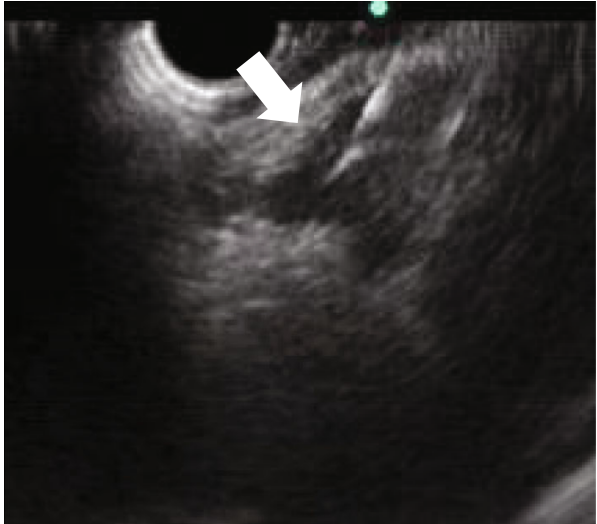

(b)

FIGURE 2: A 53-year-old male patient. (a) Left lower paratracheal lymphadenopathy (arrow) on CT scan. (b) On EUS view, triangle-shaped hypoechoic lymph node (arrow) was observed in station 4L. The EUS-guided biopsy was performed. CT: computed tomography; EUS: endoscopic ultrasound.

TABLE 4: Diagnostic yield for histologic confirmation by needle type.

\begin{tabular}{|c|c|c|c|c|}
\hline & Total $(n=69)$ & Trucut $(n=33)[95 \% \mathrm{CI}]$ & Aspiration $(n=36)[95 \% \mathrm{CI}]$ & $P$ value \\
\hline Accuracy, \% (n) & $79.7(55 / 69)$ & $84.8(28 / 33)[67.4-100]$ & $75.0(27 / 36)[47.5-100]$ & 0.310 \\
\hline Sensitivity, \% (n) & $57.6(19 / 33)$ & $66.7(10 / 15)[64.8-100]$ & $50.0(9 / 18)$ [39.9-100] & 0.335 \\
\hline Specificity, \% (n) & $100(36 / 36)$ & $100(18 / 18)[\mathrm{NA}]$ & $100(18 / 18)[\mathrm{NA}]$ & - \\
\hline PPV, \% $(n)$ & $100(19 / 19)$ & $100(10 / 10)[\mathrm{NA}]$ & $100(9 / 9)[\mathrm{NA}]$ & - \\
\hline NPV, $\%,(n)$ & $78.0(36 / 50)$ & $78.3(18 / 23)[64.5-100]$ & $66.7(18 / 27)[46.7-100]$ & 0.363 \\
\hline
\end{tabular}

CI: confidence interval; PPV: positive predictive value; NPV: negative predictive value, NA: not available.

newer end-cutting FNB needle (SharkCore ${ }^{\mathrm{TM}}$ or Acquire $\left.^{\mathrm{TM}}\right)$. Fourth, not all patients with mediastinal lymphadenopathy were attempted EUS-guided biopsy. Some patients were initially excluded from the EUSguided biopsy due to coagulopathy, use of anticoagulant, and difficult location to access by EUS. So, there would be potential of selection bias, and adverse event would be underestimated. Therefore, a randomized, controlled trial in large volume multicenter is needed to assess the efficacy and safety of EUS and EBUS in the future.

In conclusion, the EUS-guided fine needle biopsy can be a useful method for pathologic confirmation of mediastinal nodal lesions, particularly at centers in which EBUS-guided biopsy is unavailable. 


\begin{abstract}
Abbreviations
EBUS: Endobronchial ultrasound

LN: Lymph node

EUS: Endoscopic ultrasound

FNA: Fine needle aspiration

PPV: Positive predictive value

NPV: Negative predictive value

CT: Computed tomography.
\end{abstract}

\section{Data Availability}

The [DATA TYPE] data used to support the findings of this study are available from the corresponding author upon request.

\section{Conflicts of Interest}

There is no conflict of interest relevant to this manuscript.

\section{Acknowledgments}

This work was supported by the Korea Medical Device Development Fund grant funded by the Korea government (the Ministry of Science and ICT, the Ministry of Trade, Industry and Energy, the Ministry of Health \& Welfare, and the Ministry of Food and Drug Safety) (Project Numbers 1711138534 and KMDF_PR_20200901_0248-202103). The authors would like to thank Professor Jung Hwan Lee for the data collection.

\section{References}

[1] K. Yasufuku, M. Chiyo, Y. Sekine et al., "Real-time endobronchial ultrasound-guided transbronchial needle aspiration of mediastinal and hilar lymph nodes," Chest, vol. 126, no. 1, pp. 122-128, 2004.

[2] K. Adams, P. L. Shah, L. Edmonds, and E. Lim, "Test performance of endobronchial ultrasound and transbronchial needle aspiration biopsy for mediastinal staging in patients with lung cancer: systematic review and meta-analysis," Thorax, vol. 64, no. 9, pp. 757-762, 2009.

[3] P. Vilmann, P. F. Clementsen, S. Colella et al., "Combined endobronchial and esophageal endosonography for the diagnosis and staging of lung cancer: European Society of Gastrointestinal Endoscopy (ESGE) guideline, in cooperation with the European Respiratory Society (ERS) and the European Society of Thoracic Surgeons (ESTS)," Endoscopy, vol. 47, no. 6, pp. 545-559, 2015.

[4] M. F. Catalano, R. Nayar, F. Gress et al., "EUS-guided fine needle aspiration in mediastinal lymphadenopathy of unknown etiology," Gastrointestinal Endoscopy, vol. 55, no. 7, pp. 863869, 2002.

[5] R. Srinivasan, M. S. Bhutani, N. Thosani et al., "Clinical impact of EUS-FNA of mediastinal lymph nodes in patients with known or suspected lung cancer or mediastinal lymph nodes of unknown etiology," Journal of gastrointestinal and liver diseases: JGLD, vol. 21, no. 2, pp. 145-152, 2012.

[6] S. R. Puli, J. Batapati Krishna Reddy, and M. L. Bechtold, "Endoscopic ultrasound: it's accuracy in evaluating mediastinal lymphadenopathy? A meta-analysis and systematic review," World Journal of Gastroenterology: WJG, vol. 14, no. 19, pp. 3028-3037, 2008.

[7] M. F. Catalano, M. V. Sivak Jr., T. Rice, L. A. Gragg, and J. van Dam, "Endosonographic features predictive of lymph node metastasis," Gastrointestinal Endoscopy, vol. 40, no. 4, pp. 442-446, 1994.

[8] F. C. Detterbeck, M. A. Jantz, M. Wallace, J. Vansteenkiste, and G. A. Silvestri, "Invasive mediastinal staging of lung Cancer," Chest, vol. 132, no. 3, pp. 202S-220S, 2007.

[9] M. J. Wiersema, R. H. Hawes, L. C. Tao et al., "Endoscopic ultrasonography as an adjunct to fine needle aspiration cytology of the upper and lower gastrointestinal tract," Gastrointestinal Endoscopy, vol. 38, no. 1, pp. 35-39, 1992.

[10] M. J. Wiersema, P. Vilmann, M. Giovannini, K. J. Chang, and L. M. Wiersema, "Endosonography-guided fine-needle aspiration biopsy: diagnostic accuracy and complication assessment," Gastroenterology, vol. 112, no. 4, pp. 1087-1095, 1997.

[11] A. Yamabe, A. Irisawa, M. S. Bhutani et al., "Efforts to improve the diagnostic accuracy of endoscopic ultrasound-guided fineneedle aspiration for pancreatic tumors," Endoscopic ultrasound, vol. 5, no. 4, pp. 225-232, 2016.

[12] D. Oh, D. W. Seo, S. M. Hong et al., "Endoscopic ultrasoundguided fine-needle aspiration can target right liver mass," Endoscopic ultrasound, vol. 6, no. 2, pp. 109-115, 2017.

[13] M. F. Catalano, S. Sial, A. Chak et al., "EUS-guided fine needle aspiration of idiopathic abdominal masses," Gastrointestinal Endoscopy, vol. 55, no. 7, pp. 854-858, 2002.

[14] Y. N. Lee, J. H. Moon, H. K. Kim et al., "A triple approach for diagnostic assessment of endoscopic ultrasound-guided fine needle aspiration in pancreatic solid masses and lymph nodes," Digestive Diseases and Sciences, vol. 59, no. 9, pp. 2286-2293, 2014.

[15] P. J. Parekh, R. Majithia, D. L. Diehl, and T. H. Baron, "Endoscopic ultrasound-guided liver biopsy," Endoscopic ultrasound, vol. 4, no. 2, pp. 85-91, 2015.

[16] M. J. Levy, M. L. Jondal, J. Clain, and M. J. Wiersema, "Preliminary experience with an EUS-guided Trucut biopsy needle compared with EUS-guided FNA," Gastrointestinal Endoscopy, vol. 57, no. 1, pp. 101-106, 2003.

[17] S. Varadarajulu, M. Fraig, N. Schmulewitz et al., "Comparison of EUS-guided 19-gauge Trucut needle biopsy with EUSguided fine-needle aspiration," Endoscopy, vol. 36, no. 5, pp. 397-401, 2004.

[18] L. P. Berger, R. C. Scheffer, B. L. Weusten et al., "The additional value of EUS-guided Tru-cut biopsy to EUS-guided FNA in patients with mediastinal lesions," Gastrointestinal Endoscopy, vol. 69, no. 6, pp. 1045-1051, 2009.

[19] C. M. Cho, M. Al-Haddad, and J. K. Leblanc, "Rescue endoscopic ultrasound (EUS)-guided Trucut biopsy following suboptimal EUS-guided fine needle aspiration for mediastinal lesions," Gut and liver, vol. 7, no. 2, pp. 150-156, 2013.

[20] I. Storch, M. Shah, R. Thurer, E. Donna, and A. Ribeiro, "Endoscopic ultrasound-guided fine-needle aspiration and Trucut biopsy in thoracic lesions: when tissue is the issue," Surgical Endoscopy, vol. 22, no. 1, pp. 86-90, 2008.

[21] S. F. Crinò, S. Ammendola, A. Meneghetti et al., "Comparison between EUS-guided fine-needle aspiration cytology and EUSguided fine-needle biopsy histology for the evaluation of pancreatic neuroendocrine tumors," Pancreatology: official journal of the International Association of Pancreatology (IAP), vol. 21, pp. 443-450, 2021. 
[22] A. Facciorusso, S. P. Sunny, and V. Del Prete, "Comparison between fine-needle biopsy and fine-needle aspiration for EUS-guided sampling of subepithelial lesions: a meta-analysis," Gastrointestinal Endoscopy, vol. 91, no. 1, pp. 14-22.e2, 2020.

[23] A. Facciorusso, S. F. Crinò, N. Muscatiello et al., "Endoscopic ultrasound fine-needle biopsy versus fine-needle aspiration for tissue sampling of abdominal lymph nodes: a propensity score matched multicenter comparative study," Cancers (Basel), vol. 13, no. 17, p. 4298, 2021. 